\title{
CORRESPONDENCE
}

\section{T cell epitopes in SARS-CoV-2 proteins are substantially conserved in the Omicron variant}

\author{
Seong Jin Choi ${ }^{1,7}$, Dong-Uk Kim $\mathbb{D}^{2,7}$, Ji Yun Noh ${ }^{2,3}$, Sangwoo Kim $\mathbb{D}^{4}$, Su-Hyung Park $\mathbb{D}^{2}$, Hye Won Jeong ${ }^{2,5}$ and Eui-Cheol Shin $\mathbb{D}^{2,6}{ }^{\infty}$
}

(c) The Author(s), under exclusive licence to CSI and USTC 2022

Cellular \& Molecular Immunology (2022) 19:447-448; https://doi.org/10.1038/s41423-022-00838-5

In November 2021, the Omicron variant (B.1.1.529) emerged and was designated a variant of concern (VOC) by the World Health Organization. Recently, Omicron was reported to extensively escape neutralizing antibodies elicited by COVID-19 vaccination or natural infection [1-3]. However, whether Omicron evades the T cell immunity elicited by COVID-19 vaccination or natural infection remains to be elucidated. To address this issue, we analyzed the amino acid sequences of $T$ cell epitopes identified from the original SARS-CoV-2 strain (Wuhan-Hu-1) in the Omicron variant (hCoV-19/South Africa/CERI-KRISP-K032284/2021).

First, we examined 454 major histocompatibility complex (MHC) class I-restricted $\mathrm{CD}^{+} \mathrm{T}$ cell epitopes that were previously identified by activation-induced marker (AIM) assays [4]. In the Omicron variant, $88.4 \%(137 / 155)$ of epitopes from the spike protein and $98.3 \%$ (294/299) of epitopes from nonspike proteins were fully conserved (Fig. 1A), and $94.9 \%$ $(431 / 454)$ of $\mathrm{CD}^{+} \mathrm{T}$ cell epitopes were completely conserved. We performed a similar analysis with $122 \mathrm{CD}^{+} \mathrm{T}$ cell epitopes that were previously identified by a systematic analysis using peptide-MHC class I complex multimers [5]. In the Omicron variant, $88.9 \%(16 / 18)$ and $98.1 \%(102 / 104)$ of epitopes from the spike and nonspike proteins, respectively, were fully conserved, and $96.7 \%(118 / 122)$ of $\mathrm{CD}^{+} \mathrm{T}$ cell epitopes were completely conserved. We identified 19 dominant epitopes by a meta-analysis of $\mathrm{CD}^{+} \mathrm{T}$ cell epitope data from 18 studies with 852 COVID-19 convalescents [6], and all $19 \mathrm{CD}^{+}{ }^{+} \mathrm{T}$ cell epitopes were completely conserved in the Omicron variant. We also examined $280 \mathrm{MHC}$ class II-restricted $\mathrm{CD}^{+}{ }^{+} \mathrm{T}$ cell epitopes that were previously identified by AIM assays [4]. In the Omicron variant, $80.4 \%$ (74/92) and $94.7 \%(178 / 188)$ of epitopes from the spike and nonspike proteins, respectively, were fully conserved, and $90.0 \%(252 / 280)$ of $\mathrm{CD}^{+} \mathrm{T}$ cell epitopes were completely conserved. These results indicate that the majority of T cell epitopes are considerably conserved in the Omicron variant.

Next, we performed IFN- $\gamma$ intracellular cytokine staining (ICS) assays by stimulating peripheral blood mononuclear cells (PBMCs) using overlapping peptide (OLP) pools for the spike protein of the original SARS-CoV-2 strain and Omicron. PBMCs were obtained from individuals who had recovered from infection with the original SARS-CoV-2 and individuals vaccinated with BNT162b2. Among convalescent individuals, the frequency of IFN- $\gamma^{+}$cells after stimulation with the Omicron spike OLPs was $76.74 \%$ and $88.03 \%$ of the frequency after stimulation with the original spike OLPs in $\mathrm{CD}^{+}$and $\mathrm{CD}^{+}{ }^{+} \mathrm{T}$ cells, respectively, without significant differences (Fig. 1B). Among vaccinated individuals, the frequency of IFN- $\gamma^{+}$cells after stimulation with the Omicron spike OLPs was $80.42 \%$ and $83.90 \%$ of the frequency after stimulation with the original spike OLPs in $\mathrm{CD}^{+}$and $\mathrm{CD}^{+}{ }^{+} \mathrm{T}$ cells, respectively (Fig. 1B). The decrease in the $\mathrm{CD}^{+} \mathrm{T}$ cell response was significant $(P=0.03)$, but the change in the $\mathrm{CD}^{+}{ }^{+} \mathrm{T}$ cell response was not. These results demonstrate that considerable proportions $(>75 \%)$ of SARS-CoV-2 spike-specific memory $\mathrm{CD}^{+}$and $\mathrm{CD}^{+}{ }^{+} \mathrm{T}$ cells elicited by COVID-19 vaccination or natural infection recognize and respond to the Omicron spike.

Previously known VOCs, including the Beta (B.1.351) and Delta (B.1.617.2) variants, reduce the neutralizing activities of antibodies induced by COVID-19 vaccination or natural infection [7]. However, such VOCs rarely evade memory $T$ cell responses elicited by COVID-19 vaccination or natural infection [8]. In principle, VOCs can hardly evade $\mathrm{T}$ cell responses because multiple $\mathrm{T}$ cell epitopes are scattered across structural and nonstructural proteins.

COVID-19 vaccination or natural infection induces not only neutralizing antibodies but also memory $T$ cells. Virus-specific $\mathrm{CD}^{+}$and $\mathrm{CD}^{+}{ }^{+} \mathrm{T}$ cells exert antiviral functions by eliminating virus-infected cells and producing effector cytokines, leading to rapid control of viral infection and reducing disease severity [9]. Although the Omicron variant escapes neutralizing antibodies induced by COVID-19 vaccination or natural infection [1-3], our current analysis demonstrates that $\mathrm{T}$ cell epitopes are considerably conserved in the Omicron variant and that substantial proportions of memory $\mathrm{T}$ cells elicited by COVID-19 vaccination or natural infection respond to the Omicron spike. These results indicate that memory $T$ cells may provide protective immunity during reinfection or breakthrough infection with the Omicron variant.

\footnotetext{
${ }^{1}$ Division of Infectious Disease, Department of Internal Medicine, Seoul National University Hospital, Seoul, Republic of Korea. ${ }^{2}$ Graduate School of Medical Science and Engineering, Korea Advanced Institute of Science and Technology (KAIST), Daejeon, Republic of Korea. ${ }^{3}$ Division of Infectious Diseases, Department of Internal Medicine, Korea University College of Medicine, Seoul, Republic of Korea. ${ }^{4}$ Department of Biomedical Systems Informatics, Graduate School of Medical Science, Brain Korea 21 Project, Yonsei University College of Medicine, Seoul, Republic of Korea. ${ }^{5}$ Department of Internal Medicine, Chungbuk National University College of Medicine, Cheongju, Republic of Korea. ${ }^{6}$ The Center for Viral Immunology, Korea Virus Research Institute, Institute for Basic Science (IBS), Daejeon, Republic of Korea. ${ }^{7}$ These authors contributed equally: Seong Jin Choi, Dong-Uk Kim. ${ }^{凶}$ email: ecshin@kaist.ac.kr
}

Received: 2 December 2021 Accepted: 4 January 2022

Published online: 18 January 2022 


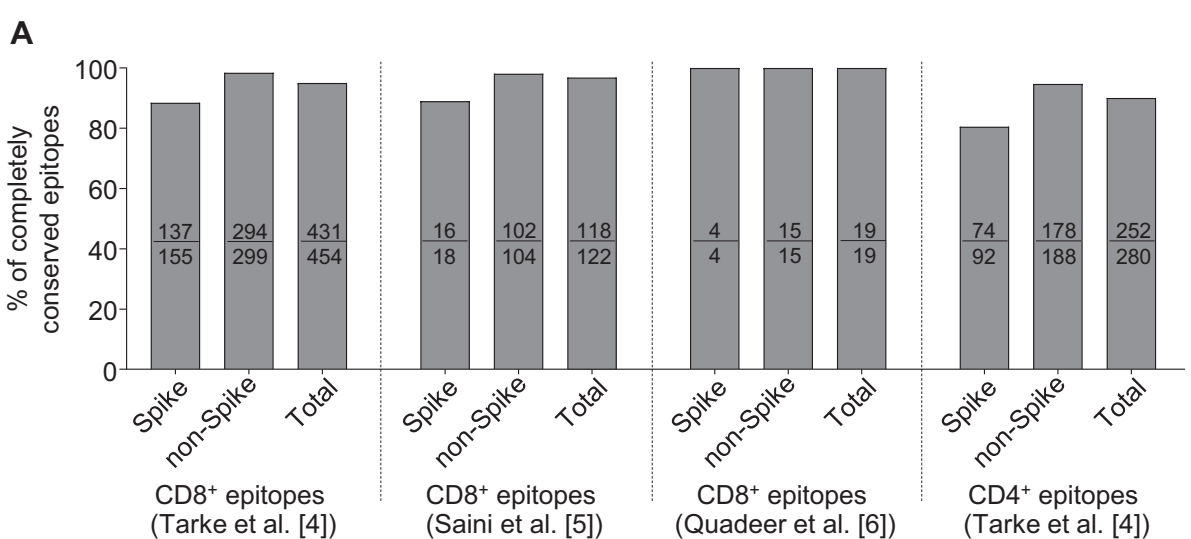

B

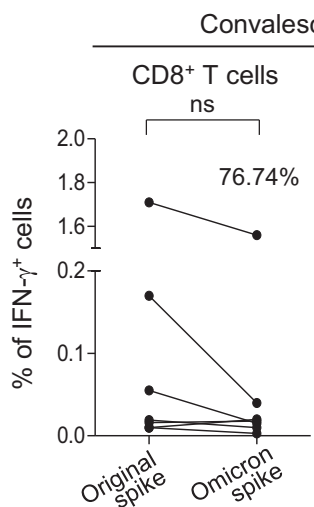

C

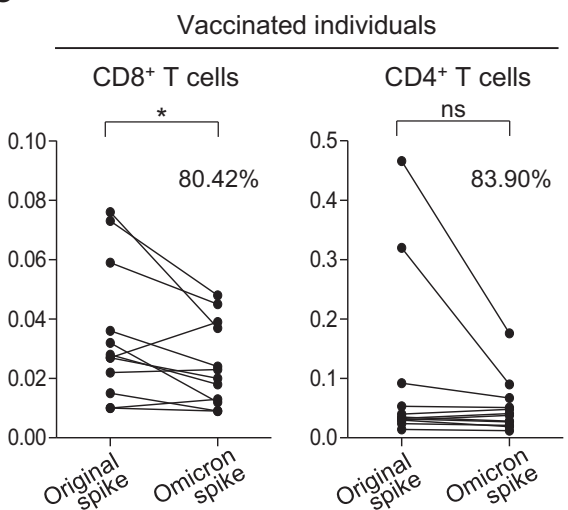

Fig. 1 T cell analysis against the Omicron variant. A Amino acid sequences of T cell epitopes identified from the original SARS-CoV-2 strain (NC_045512.2) were examined in the Omicron variant (EPI_ISL_6699770, GISAID). Four different sets of previously identified T cell epitopes were used for comparisons. Percentages of completely conserved epitopes are presented as bar graphs. B, C IFN- $\gamma$ intracellular staining was performed using PBMCs from COVID-19 convalescents $(N=7 ; 45-125$ days after symptom onset) and BNT162b2-vaccinated individuals ( $N=$ $12 ; 3$ months after the second vaccination). PBMCs were stimulated with OLP pools to spike the original strain and Omicron. The ratio of the frequency of IFN- $\gamma^{+}$cells after stimulation with the Omicron spike OLPs to the frequency after stimulation with the original spike OLPs is presented as a percentage. Statistical analysis was performed using paired $t$ tests. ${ }^{*} p<0.05$; ns not significant

\section{REFERENCES}

1. Liu L, Iketani S, Guo Y, Chan JFW, Wang M, Liu L, et al. Striking antibody evasion manifested by the Omicron variant of SARS-CoV-2. Nature. 2021. https://doi.org/ 10.1038/d41586-021-03826-3.

2. Planas D, Saunders N, Schwartz O Considerable escape of SARS-CoV-2 Omicron to antibody neutralization. Nature. 2021. https://doi.org/10.1038/d41586-021-03827-2.

3. Cele S, Jackson L, Sigal A Omicron extensively but incompletely escapes Pfizer BNT162b2 neutralization. Nature. 2021. https://doi.org/10.1038/d41586-021-03824-5.

4. Tarke A, Sidney J, Kidd CK, Dan JM, Ramirez SI, Yu ED, et al. Comprehensive analysis of T cell immunodominance and immunoprevalence of SARS-CoV-2 epitopes in COVID19 cases. Cell Rep Med. 2021;2:100204 https://doi.org/10.1016/j.xcrm.2021.100204.

5. Saini SK, Hersby DS, Tamhane T, Povlsen HR, Hernandez SPA, Nielsen M, et al. SARS-CoV-2 genome-wide $T$ cell epitope mapping reveals immunodominance and substantial CD8 + T cell activation in COVID-19 patients. Sci Immunol. 2021;6: eabf7550 https://doi.org/10.1126/sciimmunol.abf7550.

6. Quadeer AA, Ahmed SF, McKay MR. Landscape of epitopes targeted by T cells in 852 individuals recovered from COVID-19: meta-analysis, immunoprevalence, and web platform. Cell Rep Med. 2021;2:100312 https://doi.org/10.1016/j.xcrm.2021.100312.

7. Planas D, Veyer D, Baidaliuk A, Staropoli I, Guivel-Benhassine F, Rajah MM, et al. Reduced sensitivity of SARS-CoV-2 variant Delta to antibody neutralization. Nature. 2021;596:276-80. https://doi.org/10.1038/s41586-021-03777-9.

8. Tarke A. Impact of SARS-CoV-2 variants on the total CD4+ and CD8+ T cell reactivity in infected or vaccinated individuals. Cell Rep Med. 2021;2:100355 https://doi.org/10.1016/j.xcrm.2021.100355.

9. Noh JY, Jeong HW, Kim JH, Shin EC. T cell-oriented strategies for controlling the COVID-19 pandemic. Nat Rev Immunol. 2021;21:687-8. https://doi.org/10.1038/ s41577-021-00625-9.

\section{ACKNOWLEDGEMENTS}

This study was supported by the National Research Foundation Grant NRF2018M3A9D3079498 and the Institute for Basic Science (IBS), Korea, under project code IBS-R801-D2.

\section{AUTHOR CONTRIBUTIONS}

SJC, JYN, SK, S-HP, and E-CS designed the research. HWJ collected the clinical specimens. D-UK performed the experiments. SJC, D-UK, S-HP, HWJ, and E-CS analyzed the results. SJC, JYN, and E-CS wrote the manuscript.

\section{COMPETING INTERESTS}

The authors declare no competing interests.

\section{ADDITIONAL INFORMATION}

Supplementary information The online version contains supplementary material available at https://doi.org/10.1038/s41423-022-00838-5.

Correspondence and requests for materials should be addressed to Eui-Cheol Shin.

Reprints and permission information is available at http://www.nature.com/ reprints 